\title{
1. Vorbemerkung
}

Die folgenden Überlegungen stammen aus einer historisch orientierten Untersuchung zur Entwicklung der Frauenindustriearbeit in Deutschland." Die historische Orientierung verfolgt dabei eine doppelte Zielsetzung. Zum einen kann die vorfindliche Situation der Frauenerwerbstätigkeit, die gekennzeichnet ist durch die aktuellen Formen der Benachteiligung, nur aus ihrer Geschichte geklärt werden. Sie muß als Resultat einer Entwicklung begriffen werden, die trotz einer Fülle von politischen und sozialpolitischen Versuchen, die ökonomische Benachteiligung der weiblichen Arbeitskraft aufzuheben, lediglich auf jeweils neuer Ebene die Diskriminierung der Frauen im Erwerbsleben stabilisierte. Zum anderen kann so ein falscher ökonomischer Determinismus umgangen werden. Die jeweilige Verwertungssituation der Branche (Art der Produktionsprozesse und Möglichkeiten sowie Stand der Mechanisierung dieser Prozesse) ist nicht ausschließlich bedeutsam für den Fraueneinsatz. Ein weiterer zumindest gleichbedeutender Faktor ist sicher in der historischen Entwicklung der Industriezweige zu sehen: Der ursprünglich hauptsächlich durch typische weibliche Fertigkeiten und Eigenschaften gerechtfertigte Einsatz von Frauen in bestimmten Produktionsbereichen (z.B. Textil- und Bekleidungsindustrie sowie Nahrungs- und Genußmittelindustrie) besteht auch noch unter technisch entwickelteren Niveaus fort, die solche Fertigkeiten gar nicht mehr verlangen.

Wie bedeutsam die historische Entwicklung der Frauenindustriearbeit ist, wird auch im Zusammenhang mit der krisenhaften Entwicklung auf dem Arbeitsmarkt deutlich, denn die jetzige Situation auf dem Arbeitsmarkt ruft wieder verstärkt Überlegungen gegen Frauenerwerbsarbeit hervor. Die mit der Einführung der neuen Technologien verbundenen Ar* beitsmarktprobleme werden mit der Perspektive »Frauen zurück an den Herd diskutiert. Ein Abbau der Frauenerwerbsarbeit wïrde bedeuten, daß die immer knapper werdenden Arbeitsplätze Männern vorbehalten bzw. zugewiesen werden könnten. Die sozial- und familienpolitischen Überlegungen von Parteien, Gewerkschaften, aber auch männlichen Arbeitskräften, die sich sowohl in schriftlichen als auch mündlichen $\ddot{A}$ ußerungen und Diskus* sionsbeiträgen manifestieren, haben eindeutig den Tenor einer spezifischen Frauendiskriminierung. Sie versprechen sich eine Lösung der Arbeitsmarktprobleme durch eine Rückkehr der Frauen ins Haus, in die Familie. "Mutterarbeit « gilt beispielsweise in den Augen der CDU plötzlich mehr als »Erwerbsarbeit«. In dem Grundsatzpapier der Sozialausschüsse der CDU (CDA-Papier) sind etwa folgende programmatische Aussagen zu lesen:

- „Die Mutter ist unersetzlich. Ihr ist der höchste Wert anvertraut, den wir auf Erden besitzen.«

- „Die Arbeit der Mutter ist Dienst an der Gesellschaft.«

- „Mutterarbeit ist mehr als Erwerbsarbeit.«

- »Mutterarbeit führt zur Selbstverwirklichung der Frau.« 
Dabei sind solche Sätze nicht neu, sondern orientieren sich an historischen Vorläufern. Angesichts der Virulenz des Problems von Frauenerwerbsarbeit ist jedoch mehr denn je die Frage zu stellen, ob solche sozial- bzw. familienpolitischen Versuche, Frauen vom Arbeitsmarkt zu verdrängen, überhaupt zum Erfolg führen können. Wird durch die immer wiederkehrende widersprüchliche Behandlung von Frauen auf dem Arbeitsmarkt (in Zeiten von Arbeitslosigkeit den Versuch zu unternehmen, Frauen vom Arbeitsmarkt zu verdrängen; in Zeiten von Vollbeschäftigung, sie in den Arbeitsprozeß zu integrieren) historisch aufzeigbar eine Veränderung erreicht - etwa in dem Sinne, daß tatsächlich Arbeitsplätze von Frauen für Männer (denn diese sind in einer patriarchalen Gesellschaft vorrangig) bereitgestellt werden? Diese Frage impliziert jedoch eine andere, vorgelagerte: Unterliegt eigentlich Frauenerwerbsarbeit anderen, spezifischen Bedingungen im Vergleich zur männlichen Arbeit, oder besteht eine Konkurrenzsituation von männlichen und weiblichen Arbeitskräften um dieselben Arbeitsplätze? Denn nur unter der Prämisse einer tatsächlichen - nicht nur angenommenen - Konkurrenz der Geschlechter auf dem Arbeitsmarkt wären all jene historisch immer wieder aufzeigbaren Versuche, sich in Zeiten von Arbeitslosigkeit auf die »eigentliche« Rolle der Frau als Hausfrau und Mutter zu besinnen und mit Hilfe aller möglichen familien- und sozialpolitischen Maßnahmen eine Rückkehr der Frau ins Haus zu erzwingen, arbeitsmarktpolitisch überhaupt sinnvoll.

Diese vorstrukturierenden Überlegungen möchte ich im folgenden anhand einiger Aspekte des Problems der Entwicklung der Frauenindustriearbeit im historischen Verlauf deutlich machen. Im einzelnen soll beschrieben werden:

- ob und wie für den industriellen Sektor männliche und' weibliche Arbeitsbereiche sich voneinander abgrenzen lassen, d.h. also, ob Frauenindustriearbeit anderen, besonderen Bedingungen unterliegt;

- welche Auswirkungen sozial- und familienpolitische Maßnahmen in diesem Zusammenhang zeitigen.

Dabei wird sich die Darstellung auf die Zeit von der Industrialisierung (von ca. 1870, da hier zum erstenmal Daten für Deutschland vorliegen) bis zum Ende des zweiten Weltkrieges beziehen und in fünf arbeitsmarktpolitisch und historisch unterschiedlichen Phasen beschrieben werden: Die Entwicklung bis zum ersten Weltkrieg, die spezifischen Bedingun" gen während der Zeit des ersten Weltkrieges, die Entwicklung während der Weimarer Zeit sowie zum Schluß die Zeit des Dritten Reiches, die in eine Phase vor dem zweiten Weltkrieg und in eine Phase während des zweiten Weltkrieges unterteilt wird.

\section{Fraueneinsatz in der industriellen Produktion und staatlicbe Politik von 1870 bis zum Ende des zweiten Weltkriegs}

\subsection{Arbeitsteilung, Mechanisierungsbedingungen und Zuverdienerrolle als Ausgang der Femi- nisierung von Branchen $(1870$ - 1914)}

Die frühe kapitalistische Produktionsweise zeigt eine verstärkte Einbeziehung von Frauen, vornehmlich Arbeiterfrauen, in den Produktionsprozeß. Möglich wurde die hohe Beschäftigung weiblicher Arbeitskräfte durch den ausgedehnten Einsatz von einfachen und leicht zu bedienenden Maschinen und vor allem durch die Verbreitung des Prinzips der arbeits- 
teiligen Produktion (vgl. Brandt u.a., 1973, S. 21 f.; Guilbert 1966, S. 22 f.). Durch sie werden ein Großteil qualifizierter und/oder körperlich schwerer Arbeiten, die nur Männern zugänglich waren, auf eine Vielzahl körperlich leichter Arbeiten reduziert, die nur einfache Handgriffe, Geduld, Genauigkeit und Fingerfertigkeit verlangen. Dabei werden die Frauen zunächst in jene Bereiche einbezogen, die ihrer hauswirtschaftlich orientierten Ausbildung entsprechen, d.h. solche, die die industrielle Produktion der Hausarbeitstätigkeit übernehmen wie Weben, Spinnen, Nähen, Nahrungsmittel herstellen sowie Vorarbeiten für Schuster, Sattler und Bauhandwerker. ${ }^{2}$

Mit Verbreitung der Industrialisierung Ende des 19. und Anfang des 20. Jahrhunderts nimmt die Anzahl der erwerbstätigen Frauen überproportional zu (von 1882 bis 1907 um $68 \%$, dagegen die Zahl der erwerbstätigen Männer nur um 38\%). Das Verhältnis von männlichen zu weiblichen Arbeitern verändert sich von 1875 von $5,4: 1$ im Vergleich zu 1907 auf 4,7:1. 1907 sind also noch 4,7 mal mehr Männer als Frauen in der industriellen Produktion beschäftigt. ${ }^{3}$ Indessen impliziert diese Ausweitung von Frauenarbeit keine Verdrängung von männlichen Arbeitskräften. Denn zum einen bleibt der industrielle Sektor rein quantitativ eine Domäne der Männer, zum anderen bleiben die weiblichen Arbeiter im wesentlichen auf ihre traditionellen Arbeitsbereiche Textil-, Bekleidungs- und Nahrungs- und Genußmittelindustrie konzentriert. In allen drei Branchen liegt der Anteil aller Arbeiterinnen über dem der Gesamtarbeiterschaft. Über 2/3 aller Industriearbeiterinnen sind in diesen drei Branchen eingesetzt, die die traditionellen Funktionen einer geschlechtsspezifischen Arbeitsteilung implizieren. Zu Beginn des 20. Jahrhunderts gewinnen auch andere Bereiche an Bedeutung für die Frauenbeschäftigung, vor allem die Metallindustrie sowie die Industrie der Maschinen, Instrumente, Werkzeuge und Apparate. Trotz der Zunahme in den letztgenannten Industriezweigen bleiben die weiblichen Arbeiter in den drei traditionellen Bereichen überrepräsentiert, während die männlichen in allen anderen Industriezweigen anteilmäßig überwiegen (Geyer 1924).

In den Bereichen, die nicht zu den traditionellen Fraueneinsatzbereichen gehören, verrichten Frauen vornehmlich solche Tätigkeiten, die dem weiblichen Leistungsvermögen entsprechen und sich von den männlichen Arbeiten unterscheiden. Während männliche Arbeiter körperlich schwere und/oder qualifizierte Tätigkeiten - häufig in durch Mechanisierung neu entstandenen Arbeitsbereichen - übernehmen, werden den weiblichen die körperlich leichten Tätigkeiten, die sich in den meisten Fällen durch ein geringes Mechanisierungsniveau auszeichnen, zugewiesen. In allen Industriezweigen ist die Tendenz festzustellen, alle leichtere Arbeit an kleineren Gegenständen, sei es bei Maschinenbedienung oder Handarbeit, den Frauen zu übertragen. Auch räumlich arbeiten Männer und Frauen nicht zusammen. Die einzelnen Abteilungen sind entweder reine Männer- oder reine Frauenabteilungen, wobei z.B. Männer Waren erzeugen, Frauen sie sortieren, zählen und verpacken, oder Männer schwere, große Gegenstände bearbeiten, Frauen leichte und kleine, oder Män* ner qualifizierte Arbeiten ausführen, während weibliche Industriearbeiter Zuarbeiten leisten.

Trotz dieser abgrenzbaren - nur in wenigen Fällen austauschbaren - Arbeit gilt als besonderes Problem während dieser Zeit die Konkurrenz von männlichen und weiblichen Arbeitern. Der quantitativ verstärkte Einbezug von billiger Frauenarbeit führt zwar zu einer lohndrückenden Konkurrenz, diese trägt aber wiederum zu einer geschlechtsspezifischen Segmentierung auf dem industriellen Arbeitsmarkt bei. Dabei ist vor allem zu beachten, daß die geringere Bezahlung von weiblichen Arbeitern strukturelles Merkmal von Frauen- 
industriearbeit ist und ebenfalls ein Abgrenzungskriterium zu den männlichen Arbeits" kräften darstellt. Der geringe Lohn soll den Zuverdienerstatus von Frauen manifestieren, ihre Nicht-Anerkennung als vollwertige Arbeitskraft auf dem Arbeitsmarkt deutlich machen. Damit ist die Minderbezahlung der Frauen bereits an sich eine konkurrenzmildernde Maßnahme.

Die Auswirkungen dieser vielzitierten "Schmutzkonkurrenz« zeigen sich als Abwanderung von männlichen Arbeitskräften aus den Branchen Textil-, Bekleidungs- und Nahrungsund Genußmittelindustrie, wo Frauen traditionell einen hohen Anteil an den Beschäftigten haben, aber auch aus Bereichen der Metallindustrie, in die die Frauen allmählich eindringen, hin zu eher rein »männlichen Industrien«, wie Eisenindustrie, Kohlenbergbau, Baugewerbe etc. Denn hier sind die Löhne bedeutend höher als dort, wo Männer in Konkurrenz zu Frauen arbeiten. Man kann also davon ausgehen, daß überall dort, wo Frauen Arbeitsbereiche übernehmen, die Entlohnung der Arbeit tendenziell verringert wird und sinkt. Tatsächlich findet demnach keine direkte oder unmittelbare Verdrängung von Männer- durch Frauenarbeit statt, sondern es kommt vielmehr zu einer Feminisierung der sich dem weiblichen Leistungsvermögen anpassenden Branchen (Wilbrandt 1906, S. 68 f.).

So vielschichtig und wenig eindeutig wie sich die lohndrückende Konkurrenz der weiblichen Arbeitskräfte für die männlichen auf dem Arbeitsmarkt auswirkt, so wird sie auch von gewerkschaftlicher Seite aufgenommen. Obwohl es erklärtes Ziel gewerkschaftlicher Politik ist, die lohndrückende Konkurrenz von Frauen auf dem Arbeitsmarkt zu beseitigen, wird in Tarifverträgen immer wieder ein Frauenlohn festgelegt. So heißt es auf dem 2. Gewerkschaftskongreß 1896:

"Auf allen Gebieten des wirtschaftlichen Lebens mehrt sich unaufhörlich die Verwendung der weiblichen Arbeitskraft. Diese Erscheinung ist eine Folge der Tatsache, daß die Arbeiterinnen durchgängig billiger arbeiten als die Arbeiter und der kapitalistischen Ausbeutung keinen Widerstand entgegensetzen. Es ist daher für die Arbeiterklasse ein unabweisbares Gebot der Selbsterhaltung, ein Gebot der Humanität, eine Änderung dieses schmachvollen Zustandes energisch anzustreben.« (Protokoll 1896, S. 121).

Aus den vorhandenen tariflichen Vereinbarungen geht aber bis zum ersten Weltkrieg hervor, daß Frauen 30 bis $40 \%$ weniger verdienen als Männer.

Die Minderbezahlung der Frauen wird begründet mit ihrem Zuverdienerstatus, obwohl jedoch insbesondere ledige Frauen, zum Teil mit Kindern, zur Arbeit aus ökonomischen Gründen gezwungen sind.

$\mathrm{Zu}$ Beginn der Industrialisierung sind die meisten Frauen, die in die Produktion einbezogen wurden, roch unverheiratet, haben also keinen Haushalt zu versorgen. ${ }^{4}$ Erst als das Recht zur Eheschließung verallgemeinert wird und jedermann heiraten kann, gleichgültig ob er über Besitz verfügt oder nicht, werden Ehen auch im Proletariat möglich. Sie werden darüberhinaus sogar zur Ehe gezwungen, indem Gesetze gegen das Konkubinat erlassen werden und somit das Ausleben der Sexualität an die Ehe gebunden wird (vgl. Heinsohn/Knieper 1974, S. 27 f. und 76 ff.). Der Grund für diese »staatliche Förderung« des proletarischen Familienlebens liegt wohl darin, daß im aufkommenden kapitalistischen Produktionssystem immer mehr Produkte für sich ständig erweiternde Märkte produziert werden. Man benötigt also sehr viel mehr Arbeitskräfte als vorher. Die arbeitende Bevölkerung muß dementsprechend vermehrt werden. Mit der Übernahme der Lebensform der Kleinfamilie beginnt die Doppelbelastung der Frau, denn ihr allein obliegt die Führung des Haushalts und die Versorgung der Kinder zusätzlich zu ihrer Fabrikarbeit. 
Diese neu entstandene Kleinfamilie beginnt jedoch, durch die verstärkte Einbeziehung von Frauen in die Produlktion, Auflösungserscheinungen zu zeigen. Sie ist aber als gesellschaftliche Reproduktionsstätte zur Erhaltung des Arbeitskräftepotentials wie zur gesellschaftlichen Stabilisierung von besonderer Wichtigkeit. So steigt zum Beispiel die Kindersterblichkeit in Abhängigkeit von der Dauer der Berufstätigkeit der Mutter. Während bei den verheirateten Fabrikarbeiterinnen, die erst nach der Heirat in die Fabrik eintraten, 14\% der Kinder sterben, sterben bei jenen, die schon vor der Heirat Fabrikarbeiterinnen gewesen sind, $31 \%$ der Kinder im ersten Lebensjahr.

Die Verminderung der Gebährfähigkeit der Frauen, die Verwahrlosung und hohe Sterblichkeit der Kinder, ist die Folge katastrophaler Arbeitsbedingungen: Unhygienische Verhältnisse in engen, schlecht belüfteten Produktionsstätten (vor allem in den Spinnereien), kein Schutz beim Umgang mit gesundheitsschädlichen Stoffen, hohe Unfallgefahr, überlange Arbeitszeiten sowie spezifische arbeitsvertragliche Diskriminierungen (vgl. Kuczynski 1963, S. 78). Angesichts dieser Situation kommt es zu einer von den Arbeiterorganisationen initiierten Gegenbewegung, die von 1880 bis 1890 bestimmte Schutzgesetze für Frauen im Arbeitsbereich politisch durchsetzt, wie z.B. Beschränkung des Arbeitstages auf 11 Stunden, Beschäftigungsverbot für Untertagearbeit, Nachtarbeitsverbot, sowie eine Ruhezeit für vier Wochen nach der Entbindung (Thönnessen 1969, S. 45). Nach 1890 werden diese Beschäftigungsverbote erweitert.

Durch diese Schutzbestimmungen wird zum einen die reproduktive Funktion der Frauen abgesichert und zum anderen der Versuch unternommen, der »Schmutzkonkurrenz" durch eine klare Abgrenzung von weiblichen und männlichen Tätigkeiten ein Ende zu bereiten. Bestimmte Arbeitsbereiche können auf diese Weise männlichen Arbeitern vorbehalten bleiben, indem sie für weibliche verboten werden. Durch diese Ausschließung der Frauen von all jenen Tätigkeiten, bei denen der weibliche Organismus geschädigt werden könnte, wird prophylaktisch einer Auflösungserscheinung des geschlechtsspezifischen Arbeitsmarktes entgegengewirkt und eine Konkurrenz zwischen den Geschlechtern vermieden bzw. abgemildert. Der Angst, daß Frauen Männern aufgrund von spezifischen Aus" gangsbedingungen (niedrigere Reproduktionskosten, von daher geringer Lohn) von ihren Arbeitsplätzen verdrängen und zu einem Rollentausch zwingen könnten; (der Mann im Haus zuständig für Haushalt, Kindererziehung und Erhaltung der Arbeitskraft der Frau; die Frau in der Fabrik) wird damit einschränkend vorgebeugt.

Die Auswirkungen der Arbeitsschutzgesetze für die weiblichen Industriearbeiter sind dabei zwiespältig: Zwar wird der schrankenlosen Ausbeutung der Arbeitskraft Frau ein Riegel vorgeschoben, indessen verringern sich aber ihre Einsatzbereiche. Durch die Arbeitsschutzgesetze verengt sich der Arbeitsmarktzugang der Industriearbeiterinnen. Die Frauen werden zumindest partiell aus manchen Industriezweigen verdrängt (etwa den mechanisierten, in denen zur Auslastung der Maschinen auch nachts gearbeitet wird), bzw. wird ihnen der Zugang in bestimmte Arbeitsbereiche prophylaktisch verwehrt.

\subsection{Partielle Erweiterung des gescblechtsspezifischen Arbeitsmarktes (1914 - 1918)}

Wie wenig der Frauenarbeitsschutz tatsächlich von dem Schutzgedanken für die weibliche Arbeitskraft getragen, sondern von arbeitsmarktpolitischen Notwendigkeiten bestimmt wird, zeigt die Entwicklung während des ersten Weltkrieges. Arbeitsschutzgesetze 
für weibliche Industriearbeiter werden weitgehend aufgehoben; die Arbeitskraft der Frau wird erneut bis an die Grenze der physischen und psychischen Belastbarkeit ausgebeutet. Um die von Männern freiwerdenden Arbeitsplätze zu besetzen, kommt es zu einer verstärkten Einbeziehung von Frauen in die Produktion. Nach den Zusammensrellungen aus Berichten von Krankenkassen und einzelnen Industrieunternehmen ergibt sich eine Zunahme der Zahl der beschäftigten Arbeiterinnen inerhalb von zwei Jahren von 1915 - 1917 um ca. $130 \%$ (vgl. Reichsarbeitsblatt). Dabei verändert sich die Verteilung der Frauen über die einzelnen Industriezweige: Die Textil-, Bekleidungs-, Nahrungs- und Genußmittelindustrien geben ihre Vorherrschaft in der Frauenfabrikarbeit ab an die sog. Rüstungsindustrien: Metall-, Maschinen-, Elektro- und chemischer Industrie. Zwar scheint sich dadurch rein quantitativ betrachtet eine Auflösung des geschlechtsspezifisch segmentierten Arbeitsmarktes aus Ermangelung an männlichen Arbeitskräften anzudeuten, jedoch kristallisiert sich bei einer genaueren Untersuchung der Ersetzungsstrategien von Männern durch Frauen ein etwas anderes Bild heraus. Ein unmittelbarer Ersatz von gelernter Tätigkeit findet quantitativ bedeutsam hauptsächlich in den drei traditionellen Frauenbranchen statt, während die Übernahme von gelernten Tätigkeiten in den anderen Branchen $~$ vor allem der Metallindustrie - zwar überall auffindbar ist, jedoch im wesentlichen Ausnahme bleibt. In allen Branchen ist dagegen ein unmittelbarer Ersatz von an- und ungelernten Tätigkeiten häufig festzustellen, wobei jedoch bei körperlich sclıwerer Arbeit in den meisten Fällen keine unmittelbare Übernahme erfolgt.

Mittelbarer Ersatz von gelernter durch an- oder ungelernte Arbeit ist quantitativ häufiger anzutreffen als ein unmittelbarer Ersatz von Facharbeitstätigkeiten. Durch Mechanisicrung und arbeitsorganisatorische Veränderungen, insbesondere Taylorisierung der Arbeit, gelingt es, die von Frauen zu übernehmenden Arbeitsplätze zu an- bzw. ungelernten Tätigkeiten zu dequalifizieren. Ein mittelbarer Ersatz von an- und ungelernten Tätigkeiten ist für alle jene Arbeitsbereiche bedeutsam, in denen es gilt, körperlich schwere Arbeit mithilfe von Werkzeugen und Maschinen körperlich leichter zu gestalten.

Diese Beispiele zeigen, daß man nur partiell von einer Aufhebung des geschlechtsspezifischen Arbeitsmarktes sprechen kann. Denn in vielen Fällen übernehmen die weiblichen Industriearbeiter nicht die Arbeitsplätze von Männern, vielmehr werden diese Arbeitsplätze ihrer spezifischen Arbeitsleistung angepaßt.

Wie wenig Frauen trotz der quantitativen Zunahme als vollwertige Arbeitskräfte, als direkter unmittelbarer Ersatz für Männer angesehen werden, zeigt auch die Entwicklung der Löhne. Selbst wenn Frauen Männerarbeiten verrichten, bleibt der Lohn geringer. Die Spanne zwischen den Frauen- und Männerlöhnen verringert sich zwar während der Kriegszeit tendenziell; diese Angleichung kommt jedoch nur dadurch zustande, daß die Reallöhne insgesamt sinken; d.h., nicht die Frauenlöhne nähern sich den Männerlöhnen an, sondern die Männerlöhne gleichen sich den Frauenlöhnen an (Karbe 1928, S. 77 f.).

Welche Bedeutung kommt nun für diesen Zusammenhang den Frauenarbeitsschutzgesetzen zu. Es wäre verfehlt, würde man einen Zusammenhang zwischen der Aufhebung der Schutzgesetze und der partiellen Aufhebung des geschlechtsspezifisch segmentierten Arbeitsmarktes konstruieren wollen. Denn nicht die Aufhebung der Schutzgesetze zieht einen veränderten Fraueneinsatz in die industrielle Produktion nach sich, sondern die Abwesenheit von männlichen Arbeitskräften macht die Frauenindustriearbeit unumgänglich und klammert eine Konkurrenzsituation auf dem Arbeitsmarkt aus.

Deswegen sind Schutzgesetze als Mittel, männliche und weibliche Arbeitsbereiche gegenein- 
ander abzugrenzen, nicht mehr notwendig. Sie können so auf ein Minimum beschränkt werden.

Der Arbeiterinnenschutz steht während des Krieges unter dem Zeichen des Notgesetzes vom 4. August 1914, das mit den Stimmen der sozialdemokratischen Reichstagsabgeordneten verabschiedet worden ist und auch die Billigung der Gewerkschaften erhalten hat. Im $\$ 1$ des Gesetzes, betreffend Ausnahme von Beschäftigungsbeschränkungen gewerblicher Arbeiter, heißt es: „Für die Dauer des gegenwärtigen Krieges kann der Reichskanzler allgemein und für bestimmte Bezirke oder für bestimmte Arten von Anlagen, und soweit er nicht Bestimmungen erläßt, die höhere Verwaltungsbehörde für einzelne Betriebe auf Antrag Ausnahmen von den in der Gewerbeordnung vorgesehenen Beschränkungen gewähren « (Reichsgesetzblatt 1914, S. 333 f.). Hierzu treten Erlasse des Ministers für Handel und Gewerbe vom März und August 1915, welche Sonntagsarbeit für Heeresbedarf und Lebensmittelverordnung, des gleichen für den Bergbau gestatten (Leidigheit 1919, S. 27). Zunächst wird von diesem Notgesetz wenig Gebrauch gemacht. Erst mit der Umstellung von der Friedens- auf Kriegsproduktion tritt es in allen Bereichen des gewerblichen Lebens in Anwendung (vgl. Losseff-Tillmanns 1978, S. 173 f.). In allen Bezirken werden von den zuständigen Behörden Ausnahmen von den besonderen Schutzbestimmungen zugelassen, so daß sich praktisch eine Aufhebung der Arbeitsschutzbestimmungen vollzieht. Dabei handelt es sich bei den Beauftragungen meistens darum, Arbeiterinnen während der Nacht oder über die in den Gewerbeordnungen festgesetzte höchste Dauer der Arbeitszeit hinaus zu beschäftigen. Kürzungen von Pausen, Beschäftigung an Sonn- und Festtagen sind selbstverständlich. In fast allen Fällen werden die Bewilligungen ohne jeden Vorbehalt erstellt, sodaß den Unternehmern auch in bezug auf die Personen, die sie zur Überarbeit oder Nachtarbeit heranziehen wollen, freie Hand gelassen wird. Nur in einzelnen Bezirken wird vorgeschrieben, daß schwache, kränkliche, schwangere und stillende Personen nicht zur Nachtarbeit oder Überarbeit herangezogen werden dürfen.

Betrachtet man nun die Auswirkungen der Zurücknahme der Arbeitsschutzgesetze für Frauen, so ist festzustellen: es kommt zu

- einer übermäßigen Ausdehnung der Arbeitszeit, vor allem auch von Nacht- und Feiertagsarbeit;

- einer Beschäftigung von Arbeiterinnen unter gesundheitsgefährdenden Stoffen (giftige Stoffe, Gase, übermäßige Temperaturen);

- einer Beschäftigung an physisch stark belastenden Arbeitsplätzen;

- einer Beschäftigung an unfallgefährdenden Maschinen.

Somit geht die positive Funktion der Frauenschutzgesetze - Schutz ihrer reproduktiven Funktion, Schutz vor Überbelastung - verloren, während ihre negative Funktion als Mitverursacher einer Einschränkung der Frauenindustriearbeit erhalten bleibt.

\subsection{Restauration und Krise des geschlechtsspezifischen Arbeitsmarktes (1918 - 1933)}

Die Zeit nach dem ersten Weltkrieg zeigt wieder eine Konkurrenz zwischen Männern und Frauen auf dem Arbeitsmarkt. Gemäß der größeren Bedeutung von männlichen Arbeitskräften in einer patriarchalen Gesellschaftsstruktur wird versucht, die kritische Arbeitsmarktsituation zugunsten von Männern zu lösen. Unter dem Druck von staatlichen Maßnahmen sollen Frauen ihre Arbeitsplätze für die kriegsheimkehrenden Männer freimachen. Frauen sollen in der Reihenfolge folgender Dringlichkeit entlassen werden: 
- Frauen, deren Männer Arbeit haben

- alleinstehende Frauen und Mädchen

- Mädchen und Frauen, die nur 1 - 2 Personen zu versorgen haben

- alle übrigen Mädchen und Frauen (vgl. Tholen 1977, S. 26).

Zwar beschließt der Gewerkschaftskongreß 1919, daß es Pflicht der Gewerkschaft sei, bei Einstellungen und Entlassungen von Arbeitskräften frauenfeindliche Bestrebungen nicht zur Geltung kommen zu lassen, diese Verordnung bleibt jedoch nur Appell; die Betriebsräte selbst werden ein Instrument »organisierter Frauenfeindlichkeit «(vgl. Thönnessen 1969, S. 103); sie setzen häufig gegen die Unternehmen, die die eingearbeiteten »billigen Frauen« behalten wollen, Entlassungén von Frauen durch. Von staatlicher Seite werden die Entlassungen von Frauen noch durch steuerliche Begünstigungen und direkte Familienzulagen unterstützt. Es setzt ein Normalisierungseffekt des Frauenanteils in allen Branchen ein, wobei der Rückgang von weiblichen Industriearbeitern besonders stark in den Bereichen ist, die vor dem Krieg vornehmlich Männern vorbehalten waren und wo im Krieg ein Er: satz von Männer- durch Frauenarbeit stattgefunden hatte, wie im Bergbau, bei der Eisenund Metallgewinnung, der Chemischen Industrie, der Metallverarbeitung sowie der Industrie der Steine und Erden.

Während 1921 von allen Arbeiterinnen 58,1\% in den drei typischen traditionellen Frauenarbeitsbereichen Textil-, Bekleidungs- und Nahrungs- und Genußmittelindustrie beschäftigt sind und $25,7 \%$ in den oben genannten männlichen Arbeitsbereichen, verschiebt sich das Verhältnis 1924 zugunsten der »Frauenarbeitsbereiche«. Hier sind 1924 62,6\% aller Frauen beschäftigt, dagegen in den »Männerarbeitsbereichen « nur noch 22,0\% (eig. Berechnungen). Der Umstrukturierungsprozeß des Arbeitsmarktes, der im Krieg stattgefunden hat, wird damit also wieder - zumindest partiell - rückgängig gemacht. So kann man davon ausgehen, daß einige Frauen in die Familien zurückkehren, im wesentlichen aber an ihre angestammten Arbeitsplätze zurückkehren. Die Arbeiterinnenentlassungen zu Beginn der Weimarer Republik scheinen demnach ein Kampfmittel zu sein zur Regulierung des Arbeitsmarktes, wenn sie auch ihr Ziel, die Frauen vom Arbeitsmarkt zu verdrängen, nicht erreichen. Von 1924 bis 1928 stabilisiert sich der Arbeitsmarkt, die weibliche Industriearbeit nimmt wieder zu. In den weiblichen Arbeitsbereichen überproportional nur in der Nahrungs- und Genußmittelindustrie, in den männlichen Arbeitsbereichen vor allem in der chemischen Industrie.

Während der Weltwirtschaftskrise - von 1928/29 bis zum Ende der Weimarer Republik 1933 - nimmt die Beschäftigung von Frauen in den Frauenbranchen stärker ab als die von Männern, dagegen in den von Männern dominierten Bereichen insbesondere der chemischen Industrie weniger stark.

Es wird auf den weiblichen Arbeitsmarkt in dieser Krisensituation anders reagiert als auf den männlichen.

Männer werden insgesamt in größerem Umfang entlassen als Frauen. Ausnahmen bilden erstaunlicherweise jene Bereiche, in denen Industriearbeiterinnen in breiterem Umfang eingesetzt waren und sind, wie die Elektroindustrie, Feinmechanik und Optik, die Nahrungs- und Genußmittelindustrie, die Papier- und Vervielfältigungsindustrie sowie die Textilindustrie. Nur hier werden mehr Frauen als Männer entlassen.

Zwar sind diese Umstrukturierungen auf dem Arbeitsmarkt nur geringfügig und nur in den Bereichen von unqualifizierter Arbeit anzutreffen; dennoch kann man sagen, daß sich in dieser Krisensituation offensichtlich kurzfristig ökonomische Interessen an billigen Ar- 
beitskräften stärker durchsetzen können als solche, wie z.B. staatliche oder gewerkschaftliche, die das Prinzip einer branchenmäßig geschlechtsspezifischen Zuordnung wünschen. In solchen Momenten wird das Prinzip des geschlechtsspezifischen Arbeitsmarktes - soweit es sich auf eine Verteilung auf Industriezweige bezieht - löchrig.

Sieht man von der Zeit der Weltwirtschaftskrise ab, »normalisiert « sich in der Weimarer Republik die Abgrenzung von männlichen und weiblichen Arbeitsbereichen wieder. Um keine Konkurrenz aufkommen zu lassen, werden Frauen von jenen Arbeitsplätzen, die sie im Krieg übernommen hatten, die aber vor dem Krieg eine Domäne der Männer waren, verdrängt und in die Familie bzw. an ihnen "gerechte Abeitsplätze« zurückgeschickt. Durch Rationalisierungsmaßnahmen verändern sich die Arbeitsstrukturen, wobei Tätigkeiten so vereinfacht werden, daß sie von Frauen ausgeübt werden können oder müssen. Die Tätigkeiten, die Frauen verrichten, zeichnen sich durch geringe kognitive Anforderungen, jedoch hohe psychische und nervliche Belastungen aus und sind im Hinblick auf Qualifikation und Belastungen von den männlichen Arbeitsbereichen durchaus zu unterscheiden. Auch hier ist wieder festzustellen, daß Frauen nach wie vor als eine »lohndrückende Konkurrenz« arbeiten.

Ein besonderes Problem zur Zeit der Weimarer Republik ist die Abnahme der Geburtenhäufigkeit, die besonders angesichts der durch den Ersten Weltkrieg dezimierten Bevölkerung als notwendig gewordene Absicherung der biologischen Reproduktion ins Gewicht fällt. Diese Tatache sowie die Arbeitsmarktsituation (Rückkehr von Männern auf den Arbeitsmarkt und bedingt dadurch die Notwendigkeit, sowohl der Frauenindustriearbeit Grenzen zu setzen als auch die Arbeitsbereiche von Männern und Frauen zahlenmäßig und inhaltlich gegeneinander abzugrenzen) führt zu Diskussionen und Maßnahmen, die auf der einen Seite versuchen, den Bestand der Familie und der biologischen Reproduktion zu sichern, die aber auf der anderen Seite nicht die Frauenindustriearbeit abschaffen können, da sie aus ökonomischen Gründen unverzichtbar geworden ist. Hier bekommt der Frauenarbeitsschutz wieder seine Funktion: Neben den Arbeitszeitregelungen (8-StundenT'ag für alle, Nachtarbeitsverbot für Frauen etc.), die wiederum Einschränkungen für den Fraueneinsatz bedeuten, wird in der Weimarer-Republik über Mutterschutzbestimmungen verhandelt. 1926 beschließt der Reichstag, die Bestimmungen über den Mutterschutz dem Washingtoner Übereinkommen (vgl. Lossef-Tillmanns 1978, S. 274 f.) anzupassen.

Das Gesetz sieht eine Schonfrist von sechs Wochen vor und sechs Wochen nach der Niederkunft vor, sowie die Zahlung eines Entgeldes durch den Arbeitgeber, der dazu jedoch nur dann verpflichtet ist, wenn dies ausdrücklich vereinbart wurde. Weiterhin sieht das Gesetz ein Kündigungsverbot für jeweils sechs Wochen vor und nach der Niederkunft vor, wobei sich die Frist um längstens sechs Wochen verlängern kann. Unberührt davon bleiben Kündigungen, die aus einem wichtigen, nicht die Schwangerschaft betreffenden Grunde ausgesprochen werden. Das Recht, sechs Monate nach der Niederkunft Stillpausen einzulegen, ist ebenfalls gesetzlicher Bestandteil. Allerdings besteht keine Verpflichtung zur Zahlung dieser Pausen von Seiten der Arbeitgeber.

Die für eine Normalisierung des Arbeitsmarktes als unterstützende Maßnahmen vorgesehenen Familienzulagen werden zu diesem Zeitpunkt gestrichen.

Der Frauenarbeitsschutz besteht auch in der Weimarer Zeit als Regulativ zwischen der Notwendigkeit einer Begrenzung der Frauenindustriearbeit und der Notwendigkeit der Absicherung von Familie und biologischer Reproduktion. 
Die Zeit des Faschismus ist von staatlicher Seite aus von dem Versuch geprägt, weibliche Industriearbeiter vom Arbeitsmarkt zu verdrängen. Durch die Weltwirtschaftskrise ist wiederum eine verstärkte Konkurrenzsituation auf dem Arbeitsmarkt entstanden. Männer und Frauen kämpfen um eine beschränkte Anzahl von Arbeitsplätzen. Gerade die Tatsache, daß während der krisenhaften Entwicklung Ende der zwanziger Jahre mehr männliche als weibliche Arbeiter entlassen wurden, sowie die Tatsache, daß die Geburtenhäufigkeit sank und noch sinkt, führt erneut zu familienpolitischen Maßnahmen, die den Arbeiterinnen den Anreiz verschaffen sollen, an den heimischen Herd zurückzukehren. Durch Ehestandsdarlehen, Verbesserung der Schwangerschaftsberatung und Geburtshilfe, repressive Maßnahmen gegen Geburtenkontolle und Abtreibungen, Kinderbeihilfen sowie andere finanzielle und ideelle Anreize gleich zu Beginn der Machtübernahme durch die Nationalsozialisten (1933) steigt die Heirats- und Geburtenhäufigkeit (vgl. Mason 1976, S. 122 f.), und es zeigt sich gleichzeitig bis 1936 ein leichter Rückgang von Arbeiterinnen auf dem Arbeitsmarkt. Denn eine Inanspruchnahme der Ehestandsdarlehen impliziert den Verzicht auf außerhäusliche Beschäftigung. Betrachtet man jedoch die.Statistiken der damaligen Zeit genauer, so stellt man fest, daß der leichte Rückgang der Erwerbsquote der Arbeiterinnen nicht durch eine Verdrängung von Frauen - durch Männerarbeit zu erklären ist, sondern sich aus einer stärkeren Zunahme von Arbeitskräften in männlich dominierten Arbeitsbereichen ableitet.

Aufgrund der überwiegend rüstungspolitischen Orientierung der Nationalsozialisten (vgl. Sörgel 1965, S. 56) kommt es zu einem Ausbau der industriellen männlichen Arbeitsbereiche, so daß die weiblichen Arbeitskräfte zwar ihren Platz auf einem Teilarbeitsmarkt behaupten, jedoch an der Arbeitskräftezunahme nicht beteiligt sind.

Anfänglich sind zunächst einige Versuche vorhanden, Frauen durch Männer zu ersetzen. Sie gelingen jedoch nur in Ausnahmefällen. Denn in den Bereichen, in denen die Frauenarbeit besonders wichtig ist, wie in der Textil-, Bekleidungs- und Papierverarbeitenden Industrie, sowie der Elektro-, Nichteisenmetallwarenindustrie und der Feinmechanik und Optik hat sich gerade aus den älteren, verheirateten Arbeiterinnen in den Betrieben ein eingearbeiteter Stamm von Facharbeiterinnen oder angelernten Arbeiterinnen gebildet, der durch ungelernte bzw. branchenfremde männliche Arbeitskräfte nicht ohne weiteres zu ersetzen ist. Abgesehen von den Lohnvorteilen, die die weiblichen Arbeitskräfte bieten, ist vor allem die höhere Leistungsfähigkeit der Frauen nach den Berichten der Gewerbeaufsichtsbeamten dafür ausschlaggebend. Es wird festgestellt, daß z.B. in einer Hildesheimer Schraubenfabrik die Arbeitsleistung von Männern beim Ausschneiden der Schrauben und Muttern stark unter der von Frauen liegt oder daß z.B. in einer sächsischen Flachsschwingerei die Frauen einen wesentlich höheren Akkordsatz als die Männer erreichen. ${ }^{6}$ In vielen Fällen werden deshalb 1933 entlassene weibliche Arbeitskräfte kurze Zeit später wieder eingestellt.

So stößt die Familienpolitik der Nationalsozialisten an Grenzen. Die Herausbildung von frauenspezifischen Tätigkeitsbereichen verhindert eine Rückkehr von Frauen in die Familien, wirtschaftliche Not in Arbeiterfamilien gleichfalls. Sowohl das Angebot von unverheirateten Frauen, die zum Erwerb gezwungen sind, als auch die ständige Nachfrage nach weiblichen Arbeitskräften von Seiten der Unternehmen führen die Politik der Nationalsozialisten von vornherein ad absurdum. 
Als nach 1937 durch die erhöhte Rüstungsproduktion ein Mangel an Arbeitskräften einsetzt, werden die rigiden familienpolitischen Maßnahmen wieder gelockert. Es wird darauf hingewiesen, daß sich Bevölkerungswachstum und Erwerbstätigkeit von Frauen nicht ausschließen. Die Einsatzpolitik richtet sich aber weiterhin nach frauentypischen und männertypischen Arbeitsbereichen. Löhne und Arbeitsbedingungen der Frauen bessern sich dabei nicht.

Zur Abgrenzung von männlichen und weiblichen Arbeitsbereichen dient auch in dieser Zeitspanne ein besonderer Arbeitsschutz für Frauen. Während in anderen europäischen Ländern zumindest diskutiert wird, ob der Sonderschutz für Frauen nicht gegen die Gleichberechtigung verstoße, wird in Deutschland bis 1939 der Sonderschutz für Frauen bestätigt und ausgebaut. Um die Gebärfähigkeit der deutschen Frauen nicht zu gefährden, werden eine Fülle von neuen Bestimmungen erlassen. So werden bestehende Bestimmungen für schwangere und stillende Frauen ausgebaut. Die Beschäftigung von Frauen an Maschinen mit Fußantrieb wird verboten. Da die Bedienung des Fußhebels zu ständigem Stehen zwingt und somit zu einseitig das Standbein belastet, kommt es für die an den Maschinen arbeitenden Frauen zu Schäden der Wirbelsäule und der Beckenknochen sowie zu Erschütterungen der Unterleibsorgane. Das Höchstgewicht, das Frauen tragen dürfen, wird auf $15 \mathrm{~kg}$ festgelegt. Die Beschäftigung von Frauen in der Baustoffproduktion wird grundsätzlich verboten; Ausnahmen werden nur gegen Zahlung von Männerlöhnen zugelassen. So kommt es in vielen Beschäftigungsbereichen zu einer Verengung von Zugangsmöglichkeiten: Sind Maschinen mit Fußbetrieb hauptsächlich in der Leder-, Schuh- und Konservenfabrikation eingesetzt, betrifft das Verbot des Tragens eines Höchstgewichtes fast alle Einsatzbereiche. Damit ist der Frauenarbeitsschutz, wenn auch nicht in sehr wirksamer Weise, wie die quantitativen Entwicklungsdaten zeigen, so doch eine arbeitsmarktpolitische Maßnahme. Dies zeigt auch die Behandlung der Anträge im Reichsarbeitsministerium, die die Betriebe stellen. Obwohl sich nach 1936 die Anträge der Betriebe auf Ausnahmen von bestehenden Schutzvorschriften häufen, wird Anträgen nur in rüstungswirtschaftlich wichtigen Betrieben stattgegeben. Solange in der Land- und Hauswirtschaft sowie der Textilindustrie, die sich für die weibliche Erwerbstätigkeit besonders »eignen«, Mangel an Arbeiterinnen herrsche, könne man in Industrien, die sich für weibliche Arbeitskräfte wenig eigneten, die Frauenarbeit nicht erneut zulassen, heißt es in den Stellungnahmen.

Für die Frauen selbst bedeuten die Schutzgesetze so auf der einen Seite keinen wirksamen Schutz z.B. gegen überlange Arbeitszeiten und zum anderen eine Beschränkung von $\mathrm{Zu}$ gangsmöglichkeiten auf dem Arbeitsmarkt.

Die Eignung von Arbeitskräften für spezifische Arbeitsbereiche wird zum bestimmenden Kriterium eines Einsatzes. Mit Hilfe von arbeitswissenschaftlichen Untersuchungen wird wissenschaftlich abgesichert, was historisch sich längst als Realität herausgebildet hat. Frauen für frauenspezifische Tätigkeiten einzusetzen und sie abgesichert über Schutzvorschriften von anderen - männlichen Arbeitsbereichen - fernzuhalten, hat zu diesem Zeitpunkt bereits Tradition.

\subsection{Frauenindustriearbeit zwischen Bedarf und Ideologie (1939 - 1945)}

Die Bedingungen während des 2. Weltkrieges sind völlig andere als während des 1 . Weltkrieges, denn während im ersten Weltkrieg die gesamte Kriegsproduktion hauptsächlich 
mit weiblichen (deutschen) Arbeitskräften aufrechterhalten wurde, wodurch die Frauenerwerbsarbeit stark expandierte, zeigt der zweite Weltkrieg folgendes Bild: Der quantitative Einsatz von Frauen stagniert bzw. geht generell zurück. Dabei ist die Situation auf dem Arbeitsmarkt eine völlig andere als zu Beginn des ersten Weltkrieges. Im Gegensatz zu 1914 gibt es einen chronischen Mangel an Arbeitskräften und keine Arbeitslosen. Die einzige Reserve auf dem Arbeitsmarkt bilden die Frauen (vgl. Treue o.J.).

So unterscheiden sich auch die Vorbereitungen, die von staatlicher Seite für einen möglichen Kriegseinsatz getroffen werden, fundamental gegenüber jenen bei Beginn des ersten Weltkrieges.

In dem Runderlaß Nr. 426/38 Ast des Reichswirtschaftsministers werden schon 1938 Richtlinien für die Beschäftigung von Frauen im Notfall festgelegt. Danach sollen Betriebe ihren Bedarf an Arbeitskräften für den Kriegsfall berechnen und unter Einbeziehung einer zehnstündigen Arbeitszeit anmelden. Die Gewerbeaufsichtsämter werden beauftragt, festzustellen, an welchen Arbeitsplätzen Frauen anstelle von Männern beschäftigt werden können, wobei für die für Frauen geeigneten und ungeeigneten Arbeiten allgemeine Gesichtspunkte beinhalten?

- Frauen dürfen nicht mit Arbeiten beschäftigt werden, die ernsthafte Gesundheitsschädigungen mit sich bringen (z.B. Tätigkeiten mit ätzenden, giftigen und stark reizenden Stoffen sowie Gasen, gesundheitsgefährdenden Dämpfen und Staub, bei großer Hitze und Erschütterungen).

- Frauen dürfen keine solchen schweren Arbeiten übertragen werden, für die sie körperlich nicht geeignet sind.

- Frauen sollen nicht mit Arbeiten beschäftigt werden, die besondere Geistesgegenwart, Entschlußkraft und schnelles Handeln erfordern.

- Frauen sollen im allgemeinen nicht mit Arbeiten betraut werden, die besonderes technisches Verständnis und technische Kenntnisse erfordern. Der Einsatz ist aber auch hier möglich:

a) bei Frauen mit guter Auffassungsgabe nach besonderer technischer Ausbildung,

b) bei verstärkter fachkundiger Überwachung.

Man geht davon aus, daß die Beschäftigung von Frauen häufig erreicht werden kann durch Änderung der Betriebseinrichtungen oder Arbeitsverfahren (Hebe- und Fördereinrichtungen für schwere Teile, Transportbänder, selbsttätige Werkstoffzuführung, Erleichterung des Einspannens, leichte und gefahrlose Einrichtung von Maschinen, Einführung von Automaten, Aufteilung der Arbeit in einzelne, einfache Verrichtungen, Bereitstellung von Vorrichtungen und Schablonen für die Massenfertigung, fachliche Unterweisung, verstärkte Überwachung).

Man geht davon aus, daß die Beschäftigung von Frauen häufig erreicht werden kann durch Änderung der Betriebseinrichtungen oder Arbeitsverfahren (Hebe- und Fördereinrichtungen für schwere Teile, Transportbänder, selbsttätige Werkstoffzuführung, Erleichterung des Einspannens, leichte und gefahrlose Einrichtung von Maschinen, Einführung von Automaten, Aufteilung der Arbeit in einzelne, einfache Verrichtungen, Bereitstellung von Vorrichtungen und Schablonen für die Massenfertigung, fachliche Unterweisung, verstärkte Überwachung).

Die Rüstungsbetriebe, die Militärs und die zuständigen Parteipolitiker - vor allem Göring sind sich darüber im klaren, daß ein Krieg nur durch Ausnutzung aller weiblichen Arbeitskraftreserven durchgefuihrt und durchgehalten werden kann. So wird bereits vor Ausbruch 
des Krieges ein Gesetzesapparat entwickelt, der eine umfassende Dienstverpflichtung von deutschen Frauen zwischen 14 und 60 Jahren - zumindest theoretisch - erlaubt. Um nicht die Fehler des ersten Weltkrieges zu wiederholen, insbesondere um nicht die Kampfmoral der Soldaten durch schlechte Versorgung ihrer Frauen und Familien zu schwächen, wird kurz vor dem Krieg ein Gesetz zur Unterstützung der Familien entwickelt. Das Gesetz sieht vor, daß eine Unterstützung auch dann gezahlt werden soll, wenn die Frauen Einkommen haben; wobei das Einkommen allerdings nur 1/3 des Unterstïtzungssatzes ausmachen darf. Gleichzeitig besteht die Pflicht für Frauen, durch eigene Arbeit zum Unterstützungssatz beizutragen und sich bei den Arbeitsämtern zu melden. Dadurch soll vermieden werden, daß die Unterstützungsberechtigten den Arbeitsmarkt verlassen.

Im Herbst 1939 wird jedoch bereits wieder die Meldepflichtverordnung zurückgenommen. Jetzt können die Frauen, sofern sie wollen, ohne erwerbstätig zu werden, Unterstützung beziehen.

Da daraufhin tatsächlich viele Frauen ihre Erwerbsarbeit aufgeben, wird bereits im Februar 1940 wieder eine Meldepflichtverordnung sowie eine Zwangsverpflichtung für Frauen in die Rüstungsproduktion in der Parteispitze diskutiert. Nach der Meldepflichtverordnung sollen alle Frauen im Alter zwischen 15 und 40 Jahren beim Arbeitsamt, das die Einsatzfähigkeit zu prüfen hat, vorstellig werden. Die Verordnung wird jedoch nie ratifiziert. Eine Meldepflichtverordnung für Unterstützungsempfängerinnen bleibt ebenfalls lediglich in der Diskussion. Zwar ist man sich in der Parteiführungsspitze über die Notwendigkeit eines Fraueneinsatzes im klaren, aber man kann sich nicht entschließen, ihn durch $Z$ wang herbeizuführen, denn Zwang würde die Ideologie des NS-Staates in bezug auf Frauen gefährden. Der »Lebensquell« der Nation darf nicht gefährdet werden, die ideologischen Vorstellungen über Frauen als Mütter nicht durch eine Verpflichtung zur Arbeit in der Rüstung ad absurdum geführt werden, heißt es in der Parteiführungsspitze. So bleibt als einzige zulässige Maßnahme für einen verstärkten Fraueneinsatz lediglich die Werbung für einen freiwilligen Arbeitseinsatz von Frauen. Bei sich ständig verschärfender Verknappung von Arbeitskräften auf dem Arbeitsmarkt, ohne das geringste Anzeichen eines Erfolges auf die Werbekampagnen für einen freiwilligen Einsatz wird im Sommer 1941 schließlich ein Erlaß erarbeitet, der vorsieht, alle unterhaltsberechtigten Frauen, die bei Kriegsbeginn ihre Erwerbstätigkeit aufgegeben haben, zur Arbeit heranzuziehen. Nicht unter den Erlaß fallen Frauen, die nach dem September 1939 schwanger geworden sind, für pflegebedürftige Angehörige zu sorgen haben oder selbst krank sind. Um die Arbeit in der Rüstungsproduktion attraktiver zu gestalten und um dem Erlaß die Schärfe zu nehmen, soll der Verdienst aus der Erwerbsarbeit nicht mehr auf die Unterstützung angerechnet werden.

Aber auch dieser Erlaß bringt keine Entspannung auf dem Arbeitsmarkt, da die meisten Frauen Möglichkeiten finden, durch familiäre oder gesundheitliche Gründe einen Arbeitseinsatz zu umgehen. So kommt es im Januar 1943 zu einer Meldepflichtverordnung für Frauen und Männer. Danach sind alle Männer von 16 bis 65 , Frauen von 17 bis 50 Jahren meldepflichtig.

So entwickelt sich trotz der Kriegssituation ein leichter Rückgang der Frauenbeschäftigung von 1939 bis 1941 in der industriellen Produktion. Erst von 1943 bis zum Ende des zweiten Weltkrieges zeigt sich eine leichte Erhöhung. Dabei werden die weiblichen Arbeiter bis 1943 hauptsächlich in frauentypische Arbeitsbereiche eingesetzt, erst ab 1943 zeigen sich geringfügige Auflösungserscheinungen der geschlechtsspezifischen Arbeitsbereiche. Trotz des Mangels an männlichen Arbeitskräften bleibt der Versuch bestehen, sowohl die 
reproduktive Funktion der Frauen zu stützen als auch den geschlechtsspezifisch segmentierten Arbeitsmarkt nicht durcheinander zu bringen. Vielmehr ist es erklärtes Ziel nationalsozialistischer Politik, eine Konkurrenz von Männern und Frauen auf dem industriellen Arbeitsmarkt auszuschalten durch - soweit es möglich ist - eine Abgrenzung von männlichen und weiblichen Arbeitsbereichen. Zugangsbeschränkungen, die durch weiterhin bestehende Schutzvorschriften durchgesetzt werden, sowie eine ideologische Überbetonung der Mutterfunktion der Frauen wirken dabei unterstützend. So ist auch zu verstehen, daß sich Löhne und Arbeitsbedingungen für Frauen nicht verbessern.

\section{Fazit}

Betrachtet man historisch die Entwicklung von geschlechtsspezifischen Arbeitsmarktsegmenten, wird eine spezifische und unterstützende Rolle von Sozialgesetzgebung und Familienpolitik deutlich. Sozialgesetzgebung und Familienpolitik gehen von der Doppelfunktion der Frau aus und passen sich in ihrer jeweiligen Durchsetzbarkeit - von wenigen Ausnahmen abgesehen - den Gegebenheiten des Arbeitsmarktes an.

Sind familien- und sozialpolitische Maßnahmen darauf abgestellt, Frauen vom Arbeitsmarkt zu verdrängen, so gelingen solche Maßnahmen immer nur partiell. Sie vermögen lediglich zu einer Wiederherstellung der geschlechtsspezifischen Verteilung von Arbeitsplätzen und einer Normalisierung des Frauenanteils beizutragen. Denn Frauenindustriearbeit unterliegt anderen und besonderen Bedingungen. So können die Maßnahmen immer nur zur Unterstützung und Absicherung einer Abgrenzung von männlichen und weiblichen Arbeitsbereichen dienen, jedoch nicht selbst initiativ eingreifen.

Dabei ist die Familienpolitik in ihren Auswirkungen auf die Frauenlohnarbeit eindeutig diskriminierend. Indem sie die Doppelfunktion der Frau als Grundlage ihrer Maßnahmen nimmt, verhindert sie eine kontinuierliche Integration der Frau in die Berufswelt und impliziert damit Chancenungleichheit durch Anerkennung der Frau als disponibler Arbeitskraft.

Die Sozialgesetzgebung ist dagegen in bezug auf die Reduzierung von ökonomischer Diskriminierung durchaus ambivalent. Auf der einen Seite bleiben durch die Arbeitsschutzbestimmungen für die Frauen nur solche Bereiche, die den gesetzlichen Anforderungen genügen und schränken dadurch die Frauenerwerbsarbeit ein; auf der anderen Seite schützen sie die weibliche Arbeitskraft vor allzu großer Ausbeutung und sichern deren reproduktive Funktionen ab. Betrachtet man die Arbeitsschutzgesetze unter dem Blickwinkel einer Einschränkung auf die Frauenarbeit, so ist zu sagen:

- Das Verbot von physisch schwerer Arbeit verdrängt die weibliche Arbeitkraft aus jenen Bereichen industrieller Produktion, die durch körperlich schwere Tätigkeiten gekenn* zeichnet sind. Beispiele: Da die Frauen zumeist über eine schlechte Ausbildung verfügen, als ein Ergebnis der gesellschaftlichen Normierung, die die Frau auf die Familie und die Reproduktion festlegt, stehen ihrem Einsatz nur solche Bereiche zur Verfügung, die einfache Qualifikationen erfordern und/oder deren Mechanisierungsgrad physisch weniger schwere Tätigkeiten zuläßt; es sind dies insbesondere die Prozeßbereiche Montage, Verpackung und Stoffverformung, soweit hier Mechanisierungslücken bestehen. Durch diese Beschränkung ist gleichzeitig eine Konzentration auf wenige Branchen vorgezeichnet, deren Bewegung noch nicht abgeschlossen ist. 
- Das Verbot der Nachtarbeit ist mit einer Reduktion der Frauenarbeit in jenen Bereichen verbunden, die durch den Einsatz kapitalintensiver Produktionstechnik nur rentabel im Dreischichtbetrieb zu betreiben sind. Es sind dies zum Teil Branchen, in denen Frauen traditionellerweise beschäftigt waren, wie die Textil- und Bekleidungsindustrie oder die Nahrungs- und Genußmittelindustrie. Damit erfahren die Arbeitsplatzalternativen eine weitere Beschränkung.

- Mutterschutz, Hausarbeitstag etc. bedeuten für den Unternehmer zusätzliche Kosten und verhindert dadurch Aufstiegsmöglichkeiten für die Frauen.

Zusammenfassend läßt sich feststellen, daß die Interessenkonstellationen von Kapital und Staat jeweils unterschiedlich zum Tragen kommen. Schutzgesetze werden erst dann möglich, wenn sie bevölkerungspolitisch notwendig erscheinen und dann wieder abgeschafft, wenn die Bedingungen des Arbeitsmarktes es erfordern.

Die Familienpolitik (Familienideologie) sichert die biologische Reproduktionstätigkeit und damit den Fortbestand der Gesellschaft, ebenso wie die Gratisarbeit im Haushalt. Sie wirkt immer dort, wo die Alternative zur Hausarbeit fehlt und der Arbeitsmarkt keine Erwerbsbeteiligung von Frauen erfordert oder zuläßt. Eine Ausnahme stellt hier die NS-Zeit dar (vgl. Mason 1976, S. 122 f.), wo trotz Erfordernissen des Kapitals aufgrund familienpolitischer Maßnahmen auf eine Erwerbsbeteiligung von Frauen verzichtet wurde. Die Familienpolitik ist in bezug auf die ökonomische Benachteiligung von Frauen eindeutig diskriminierend.

Dagegen ist die Sozialgesetzgebung in ihren Folgen auf die Diskriminierung der Frauen im Erwerbsleben ambivalent. Zwar dient auch sie der Absicherung der biologischen Reproduktion, sie gewährleistet aber auch einen gewissen Schutz der weiblichen Arbeitskraft. Durch Beschränkung von Arbeitsmöglichkeiten für Frauen wirkt sie jedoch gleichzeitig diskriminierend.

\section{Anmerkungen}

1 Die als Dissertation angelegte Studie entstand aus einem Forschungsprojekt des Soziologischen Forschungsinstituts (SOFI) Göttingen zur strukturellen Benachteiligung von Frauen im industriellen Produktionsprozeß. Als Ergebnis weist die Dissertation, ausgehend von einer kritischen Auseinandersetzung mit dem Ansatz des spezifisch weiblichen Arbeitsvermögens, die Notwendigkeit zur Abgrenzung eines frauenspezifischen Arbeitsmarktsegments nach. Der Begriff frauenspezifisches Segment beinhaltet dabei, daß die Betriebe Frauen nicht lediglich als eine Arbeitskräftegruppe mit unspezifischen Voraussetzungen neben anderen (Ausländer, Ungelernte, Behinderte, Jungarbeiter) beschäftigen, sondern gezielt auf bestimmte Eigenheiten des weiblichen Arbeitsvermögens zurïckgreifen. So ist Frauenindustriearbeit immer besonders und von anderen vor allem männlichen - Arbeitskräftegruppen abgrenzbar. Aus diesem Projektzusammenhang wird noch ein weiteres Ergebnis hier dargestellt. Vgl. den Aufsatz von L. Lappe, „Frauen im Ghetto. Der frauenspezifische Arbeitsmarkt und seine Folgen (im gleichen Heft).

2 Vgl. H. Goos, 1970, S. 5 ff. Goos belegt diese seit einiger Zeit insbesondere von den Berliner Frauengruppen diskutierte These vor allem mit Bebel. Es wird darauf hingewiesen, daß die Arbeitsplätze nicht geschlechtsspezifisch zugewiesen wurden, sondern daß die ersten Industriezweige hauptsächlich Textilbetriebe und Nahrungs- und Genußmittelbetriebe waren, die die in größerer Anzahl auf dem Arbeitsmarkt vorhandenen Frauen einbezogen.

3 Eigne Berechnungen nach den Berufszählungen von 1882 und 1907. 
41895 waren von 1000 Fabrikarbeiterinnen 215 verheiratet (allerdings hatten auch verwitwete Frauen und unverheiratete Mütter einen Haushalt zu besorgen). Vgl. L. Braun 1901, S. 283. Vgl. auch Bebels Zahlen für das Land Baden, wo 1899 etwa 1/3 aller Arbeiterinnen verheiratet waren. A. Bebel 1891, 9. Aufl., S. 257.

5 Vgl. R. Wilbrandt 1906, S. 47. Wilbrandt stützt sich bei seiner Darstellung auf die Berichte von Mainzer Fabrikinspektoren gegen Ende des 19. Jahrhunderts.

6 Die Beispiele sind zusammengestellt bei D. Winkler 1977, S. $43 \mathrm{ff}$.

7 Vgl. U. v. Gersdorff 1969, S. 285 ff., die eine umfangreiche Quellensammlung zusammengestellt hat.

\section{Literatur}

A. Bebel, Die Frau und der Sozialismus, Stuttgart 1891, 9. Aufl.

G. Brandt, I. Kootz, G. Steppke, Zur Frauenfrage im Kapitalismus, Frankfurt/M. 1973

L. Braun, Die Frauenfrage. Ihre geschichtliche Entwicklung und ihre wirtschaftliche Seite, Leipzig 1901

U. v. Gersdorff, Frauen im Kriegsdienst 1914 - 1945, Stuttgart 1969

A. Geyer, Die Frauenerwerbsarbeit in Deutschland, Jena 1924.

H. Goos, Einflïsse des technischen Fortschrittes auf Frauenarbeit in der Industrie (dargestellt am Beispiel eines Verpackungsbetriebes in der pharmazeutischen Industrie), Magisterarbeit, Göttingen 1970

M. Guilbert, Les Fonctions des Femmes dans l'industrie, Paris 1966.

Vgl. G. Heinsohn, R. Knieper, Theorie des Familienrechts. Geschlechtsrollenaufhebung, Kindervernachlässigung, Geburtenruickgang, Frankfurt/M. 1974.

A. Karbe, Die Frauenlohnfrage und ihre Entwicklung in der Kriegs- und Nachkriegszeit, Rostock 1928.

J. Kuczynski, Die Geschichte der Lage der Arbeiter unter dem Kapitalismus, Band 18, Berlin (Ost) 1963.

H. Leidigheit, Die Fabrikarbeit verheirateter Frauen, Dissertation, Greifswald 1919.

G. Losseff-Tillmanns, Frauenemanzipation und Gewerkschaften, Wuppertal 1978.

I. Mason, Zur Lage der Frauen in Deutschland 1930 bis 1940: Wohlfahrt, Arbeit und Familie, in: Gesellschaft, Beiträge zur Marxschen Theorie, Frankfurt/M. 1976.

Protokoll des 2. Gewerkschaftskongresses 1896.

Reichsarbeitsblatt 1914 - 1918, Berichte der Krankenkassen

Reichsgesetzblatt 1914

W. Sörgel, Metallindustrie und Nationalsozialismus, Frankfurt/M. 1965.

W. Thönnessen, Frauenemanzipation, Politik und Literatur der deutschen Sozialdemokratie zur Frauenbewegung 1863 - 1933, Frankfurt/M. 1969.

'M. Tholen, Zur Entwicklung der industriellen Frauenarbeit in der Weimarer Republik und der Arbeiterinnenpolitik der Gewerkschaften, unveröffentl. schriftl. Hausarbeit zur Diplomprïung, Bielefeld 1977.

W. Treue, Wirtschaft und Politik 1933 - 1945. Beiträge zur Geschichte der jüngsten Vergangenheit, Heft 4, Hannover o.J.

Wilbrandt, R., Die Frauenarbeit. Ein Problem des Kapitalismus, Leipzig 1906.

D. Winkler, Frauenarbeit im Dritten Reich, Hamburg 1977. 